\title{
Diffusion tensor MR imaging (DTI) metrics in the cervical spinal cord in asymptomatic HIV-positive patients
}

\author{
Christina Mueller-Mang • Meng Law • Thomas Mang • \\ Julia Fruehwald-Pallamar • Michael Weber • \\ Majda M. Thurnher
}

Received: 8 August 2010 / Accepted: 13 October 2010/Published online: 3 November 2010

(C) The Author(s) 2010. This article is published with open access at Springerlink.com

\begin{abstract}
Introduction This study was conducted to compare diffusion tensor MR imaging (DTI) metrics of the cervical spinal cord in asymptomatic human immunodeficiency virus (HIV)-positive patients with those measured in healthy volunteers, and to assess whether DTI is a valuable diagnostic tool in the early detection of HIV-associated myelopathy (HIVM).

Methods MR imaging of the cervical spinal cord was performed in 20 asymptomatic HIV-positive patients and in 20 healthy volunteers on a 3-T MR scanner. Average fractional anisotropy (FA), mean diffusivity (MD), and major (E1) and minor (E2, E3) eigenvalues were calculated within regions of interest (ROIs) at the $\mathrm{C} 2 / 3$ level (central and bilateral anterior, lateral and posterior white matter).

Results Statistical analysis showed significant differences with regard to mean E3 values between patients and controls $(p=0.045$; mixed-model analysis of variance (ANOVA) test). Mean FA was lower, and mean MD, mean E1, and mean E2 were higher in each measured ROI in patients compared to controls, but these differences were not statistically significant.

Conclusion Asymptomatic HIV-positive patients demonstrate only subtle changes in DTI metrics measured in the
\end{abstract}

C. Mueller-Mang $\cdot$ T. Mang $\cdot$ J. Fruehwald-Pallamar $\cdot$ M. Weber $\cdot$

M. M. Thurnher $(\triangle)$

Department of Radiology, Medical University of Vienna,

Waehringer Guertel 18-20,

1090 Vienna, Austria

e-mail: Majda.thurnher@meduniwien.ac.at

M. Law

Department of Radiology, Keck School of Medicine,

University of Southern California,

Los Angeles County Hospital and USC Medical Center,

Los Angeles, CA, USA cervical spinal cord compared to healthy volunteers that currently do not support using DTI as a diagnostic tool for the early detection of HIVM.

Keywords Diffusion tensor imaging .

Human immunodeficiency virus-associated myelopathy .

Spinal cord · Vacuolar myelopathy

\section{Introduction}

Human immunodeficiency virus (HIV)-associated myelopathy (HIVM) and acquired immunodeficiency syndrome (AIDS)-associated myelopathy are terms used for HIVrelated spinal cord abnormalities. Although HIV penetration of the central nervous system (CNS) occurs in the early stages of HIV infection, HIVM usually manifests clinically in advanced stages of disease, and often in conjunction with HIV encephalopathy [1]. The histopathologic correlate in most of these patients is vacuolar myelopathy (VM), which has been found in up to $46.5 \%$ in autopsy studies on patients with AIDS, but is frequently unrecognized during life [2]. The clinical diagnosis of VM is suspected in cases of slowly progressive lower extremity weakness, gait disorder, and sphincter and erectile dysfunction in an HIV-positive individual [3].

The diagnosis of HIVM remains challenging with conventional MR imaging sequences. High signal intensity abnormalities, without cord expansion or enhancement, as well as spinal cord atrophy, have been described in a limited number of cases $[4,5]$. Vacuolar myelopathy is a diagnosis of exclusion, with MR imaging currently mainly performed to exclude infectious, degenerative, or neoplastic disorders.

Diffusion tensor MR imaging (DTI) has recently shown promising results for the detection of early myelopathic 
changes in the setting of cervical spondylotic myelopathy (CSM) $[6,7]$ and in patients with spinal cord trauma [8]. There is also preliminary evidence that suggests increased sensitivity of DTI for the detection of otherwise occult spinal cord pathology in multiple sclerosis $[9,10]$.

The purpose of this study was to evaluate the ability of DTI metrics for the detection of HIVM. The hypothesis was that, given the early pathologic changes, DTI may be able to detect alterations in the normal-appearing cervical spinal cord in HIV-positive patients that precede the detectable spinal cord changes associated with HIVM. Considering the predilection of VM for the lateral and posterior columns of the spinal cord, we used axial DTI for measurement of focal regions corresponding to the known location of various white matter tracts, and investigated whether the changes in DTI metrics correlated with the distribution of VM.

\section{Material and methods}

\section{Subjects}

In this prospective study, 20 asymptomatic HIV-positive patients ( 15 men and five women), ranging in age from 25 to 74 years (mean age, 45 years), who were referred for MR imaging from the HIV unit between March 2007 and February 2008, were included. Only patients without clinical signs of myelopathy due to HIV or causes other than HIV were selected. The CD4 cell count was measured in all patients within 1 month of the time of MR imaging. Ten patients had a CD4 cell count of less than 200 cells $/ \mathrm{mm}^{3}$ and could therefore be classified as having AIDS according to the Centers for Disease Control and Prevention [11].

Twenty healthy volunteers (nine men and 11 women) with a mean age of 42 years (range, 25-77 years), and without any known spinal disease or neurologic disorder, were also recruited.

Four patients and five controls with signs of severe cervical spondylosis on MR imaging were excluded from the study.

The study was conducted with the approval and in accordance with the regulations of the local institutional ethics board, and written informed consent was obtained from all of the subjects before data acquisition.

\section{MR imaging technique}

All patients and control subjects underwent MR imaging on a 3-T MR imaging scanner (Magnetom Tim Trio, Siemens Medical Solutions, Erlangen, Germany). Using standard volume neck coils, the following pulse sequences were acquired: localizing sagittal and coronal T1-weighted images, followed by turbo spin-echo sagittal T1-weighted $(\mathrm{TR} / \mathrm{TE}, 700 / 11 \mathrm{~ms}$; matrix $256 \times 92)$ and sagittal T2- weighted (TR/TE, 3210/110 ms; matrix $256 \times 93)$ images. A 3-mm section thickness and a $220-\mathrm{mm}$ field of view were used. These sequences were performed in order to exclude spinal cord lesions and to enable the acquisition of an axial DTI corresponding to the level $\mathrm{C} 2 / \mathrm{C} 3$. None of the patients received intravenous contrast material. Axial DTI of the upper cervical spinal cord was then performed using pulsed gradient, spin-echo, double-shot, echo-planar imaging with an acceleration factor of two and six non-collinear gradient directions applied with one $b$ value $\left(b=1000 \mathrm{~s} / \mathrm{mm}^{2}\right.$; field of view, $180 \times 180 \mathrm{~mm}$; image matrix, $256 \times 256$; ten contiguous 3-mm sections; TR/TE, 3700/98 ms). The DTI imaging plane was parallel to the conventional axial images, and perpendicular to the long axis of the spinal cord. The duration of DTI acquisitions was slightly over 2 min per patient study, during which time the patients were asked to hold still and to try to avoid swallowing. Images were obtained in only six directions to reduce the scan acquisition time. Increasing the number of directions increases the scan time, which makes the acquisition prone to physiologic motion artifacts. For anatomic comparison, axial turbo spinecho T2-weighted images (TR/TE, 3484/120 ms) of the upper cervical spine (levels $\mathrm{C} 2$ to $\mathrm{C} 4$ ) were obtained. In addition, every patient underwent MR imaging of the brain on the same MR scanner using standardized head coils and the following sequences: axial FLAIR-weighted images, coronal T2-weighted images, and axial T1-weighted images.

\section{Imaging processing}

The diffusion tensor images were transferred to a dedicated workstation (Leonardo, Siemens Medical Solutions, Erlangen Germany), where the data were post-processed using the DTI module included in the Syngo ${ }^{\circledR}$ VX49B imaging software platform. Mean diffusivity (MD) and fractional anisotropy (FA), as well as the major (E1) and minor eigenvalues (E2-E3), were calculated from the DTI metrics on a voxel-by-voxel basis and displayed as twodimensional color and gray-scale images. From these images, the average metric values were measured in specific regions of interest (ROIs) according to a technique previously described [9]. For each patient, FA, MD, and E1-E3 were measured at the level of the $\mathrm{C} 2 / \mathrm{C} 3$ disc space in ROIs placed at the anterior, lateral, and posterior regions of the spinal cord, with separate, bilateral ROIs at each of these positions (Fig. 1). Special care was taken to avoid cerebrospinal fluid (CSF) partial volume effects and magnetic susceptibility and motion artifacts in the selection of each ROI. The anterior and posterior ROIs were placed in a paramidline location to approximate the anterior and posterior columns. The lateral ROIs were placed to approximate the location of the corticospinal tracts. A seventh, slightly larger ROI was placed centrally within the 


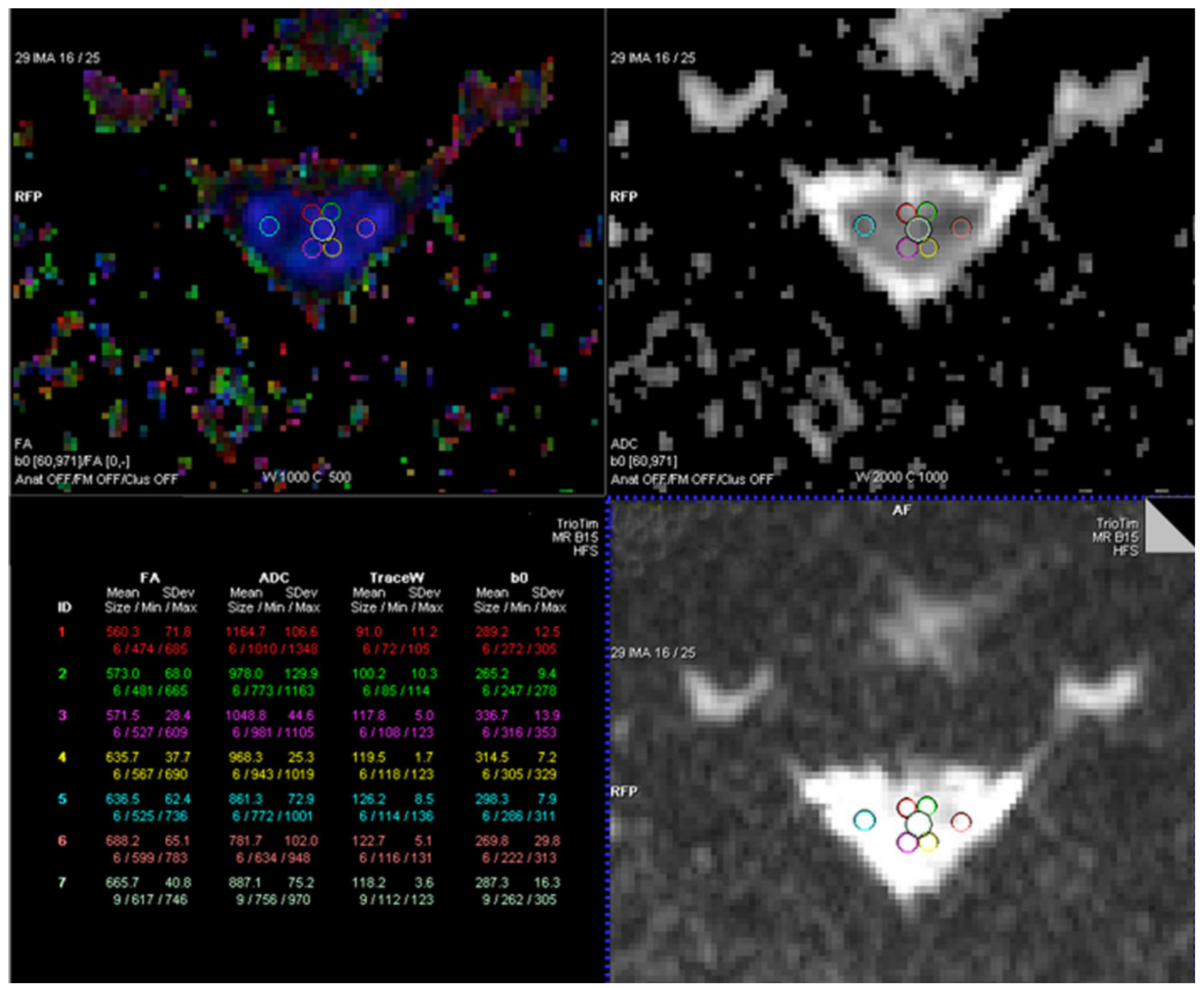

Fig. 1 FA map and corresponding ADC map and B0 image showing ROIs placed in the central, and bilateral anterior, lateral, and posterior spinal cord at the $\mathrm{C} 2-\mathrm{C} 3$ level. The table on the left shows the

cord, covering the expected location of both gray and white matter. The ROIs were manually set on the FA images, and then copied to the other created DTI maps. In order to be able to accurately place the ROIs within the expected locations of anterior, posterior and lateral white matter, the size of the ROIs had to be adapted to the axial size of the spinal cord which was differing between various patients. Therefore, the average areas of ROIs in the anterior, posterior, and lateral spinal cord measured between three and seven pixels, while the centrally located ROI measured between seven and 12 pixels.

Statistical analysis

The data for each patient and control subject consisted of one $\mathrm{FA}$, one $\mathrm{MD}$, one $\mathrm{E} 1$, one $\mathrm{E} 2$, and one $\mathrm{E} 3$ measure within each of seven spinal cord ROIs at the $\mathrm{C} 2-\mathrm{C} 3$ level automatic calculation of mean value, standard deviation, size, and minimum and maximum value of the different ROIs

(central, right and left anterior, right and left posterior, right and left lateral). Each measure represented the average overall voxels within the respective region of interest. For statistical purposes, the separate measurements from each side were merged into one value, resulting in four ROIs (anterior, posterior, lateral, central) for each DTI metric in patients and controls. A mixed-model analysis of variance (ANOVA) test was performed to determine whether any significant differences in DTI metrics were found among the various regions between patients and controls. For the comparison of patients and controls for each region separately, unpaired $t$-tests were used as post hoc tests. Due to the small sample size, no corrections were performed in order to avoid an increasing type 2 error.

All statistical calculations were performed with SPSS software (SPSS version 16.0; SPSS, Chicago, IL), and $p$ 
values of equal to or less than 0.05 were considered statistically significant.

\section{Results}

None of the patients and healthy volunteers showed any spinal cord abnormality on conventional MR imaging, and there were no signs of spinal canal stenosis. In addition, MR imaging of the brain did not show any HIV-related abnormalities in any patient.

Mean FA was lower and mean MD was higher in each measured ROI in patients compared with control subjects (central, right and left anterior, right and left posterior, right and left lateral), but did not reach statistical significance with the mixed-model ANOVA test $(p=0.315$ and $p=0.103$, respectively). Also, when comparing the FA and MD metrics of different regions separately, there was no significant difference between patients and controls (Table 1).

An analysis of variance did not show any significant differences between patients and controls with regard to the E1 and E2 values ( $p=0.255$ and $p=0.229$, respectively). However, there was a significant difference with regard to E3 values between patients and control subjects $(p=0.045$; mixedmodel ANOVA test). The post hoc unpaired $t$-test showed that significant differences were present only in the lateral ROI $(p=0.013)$. The mean E3 values in this region were significantly higher in patients compared to control subjects ( $E 3=0.368$ and 0.278 , respectively). A line chart of the $E 3$ values in patients and healthy volunteers is shown in Fig. 2.
Regarding the lateral ROIs in detail, there was also a trend toward lower FA values $(p=0.073)$ and higher MD and E2 values in patients compared to controls $(p=0.063$ and $p=0.083$, respectively).

Comparing mean FA values of patients with CD4 cell count below to patients with CD4 cell count above 200 cells $/ \mathrm{mm}^{3}$ the mixed-model ANOVA test did not reveal any significant difference $(p>0.05)$.

\section{Discussion}

DTI has been used extensively in the brain; however, information on the clinical utility of DTI in the spinal cord is presently limited $[7,9,12]$. This paucity of data is mainly due to the technical challenges associated with spinal cord DTI, such as the spinal cord's small size, motion artifacts related to the CSF and patient swallowing, and field inhomogeneities, particularly at 3-T field strength and above $[13,14]$. Some of these technical difficulties have been overcome by the development of faster imaging sequences and by the increasing availability of 3-T MR scanners that provide parallel imaging with improved phased array coil technology, which alleviates some of the artifacts associated with DTI of the spinal cord at $3 \mathrm{~T}$ compared to $1.5-\mathrm{T}$ MR scanners $[14,15]$.

A large number of studies on HIV encephalopathy have been published in the literature, but involvement of the spinal cord in HIV-positive patients is less well understood and documented. The frequency of HIVM varies in
Table 1 Average fractional anisotropy, mean diffusivity, and mean E1, E2, and E3 in ROIs in the anterior, posterior, lateral, and central spinal cord at the C2-C3 level, in 20 patients with HIV and 20 healthy volunteers (unpaired $t$-tests)

\begin{tabular}{lllll}
\hline Fractional anisotropy & Anterior & Posterior & Lateral & Central \\
\hline Patients & $0.581 \pm 0.107$ & $0.731 \pm 0.087$ & $0.68 \pm 0.078$ & $0.732 \pm 0.097$ \\
Controls & $0.604 \pm .056$ & $0.749 \pm 0.076$ & $0.72 \pm 0.085$ & $0.734 \pm 0.081$ \\
$P$ values & 0.382 & 0.487 & 0.073 & 0.954 \\
Mean diffusivity $\left(\times 10^{-3} \mathrm{~mm}^{-2} \mathrm{~s}^{-1}\right)$ & & & \\
Patients & $0.975 \pm 0.173$ & $0.967 \pm 0.097$ & $0.864 \pm 0.113$ & $0.914 \pm 0.107$ \\
Controls & $0.908 \pm 0.104$ & $0.915 \pm 0.147$ & $0.792 \pm 0.125$ & $0.875 \pm 0.148$ \\
$P$ values & 0.149 & 0.198 & 0.063 & 0.335 \\
E1 & & & & \\
Patients & $1.691 \pm 0.182$ & $1.983 \pm 0.176$ & $1.670 \pm 0.116$ & $1.858 \pm 0.176$ \\
Controls & $1.623 \pm 0.204$ & $1.909 \pm 0.262$ & $1.620 \pm 0.213$ & $1.803 \pm 0.279$ \\
$P$ values & 0.275 & 0.301 & 0.355 & 0.456 \\
E2 & & & & \\
Patients & $0.720 \pm 0.241$ & $0.551 \pm 0.153$ & $0.554 \pm 0.169$ & $0.534 \pm 0.155$ \\
Controls & $0.659 \pm 0.088$ & $0.536 \pm 0.139$ & $0.469 \pm 0.129$ & $0.497 \pm 0.144$ \\
$P$ values & 0.287 & 0.533 & 0.082 & 0.446 \\
E3 & & & & \\
Patients & $0.514 \pm 0.173$ & $0.374 \pm 0.112$ & $0.368 \pm 0.101$ & $0.351 \pm 0.123$ \\
Controls & $0.426 \pm 0.111$ & $0.308 \pm 0.137$ & $0.278 \pm 0.118$ & $0.319 \pm 0.123$ \\
$P$ values & 0.063 & 0.100 & 0.013 & 0.429 \\
\hline & & & &
\end{tabular}


E3 values

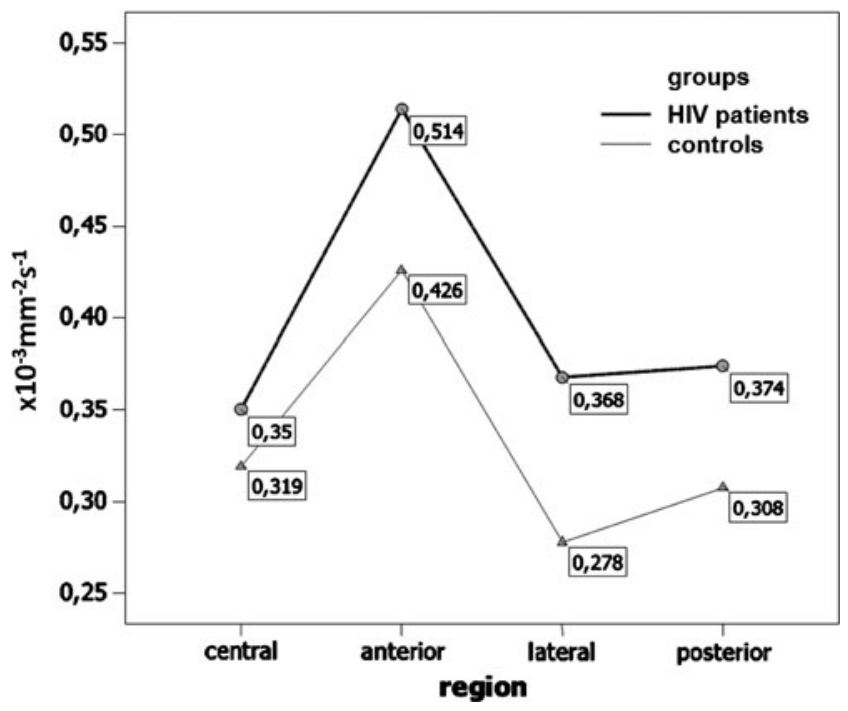

Fig. 2 Line chart of minor E3 eigenvalues in HIV-positive patients and healthy volunteers within ROIs placed in the central, anterior, posterior, and lateral spinal cord at the $\mathrm{C} 2-\mathrm{C} 3$ levels. Minor E3 eigenvalues are higher in all ROIs in HIV-positive patients compared to healthy volunteers, with a significant difference in the lateral ROI $(p=0.013$; unpaired $t$-test)

different published clinical series, from 20 to $55 \%$ [2, 1619]. The predominant pathologic feature in these patients is VM. VM is characterized by vacuolation of myelin sheaths, and was initially described by Petito et al. in 1985 [20] in AIDS patients with a previously unknown progressive myelopathy [20]. Pathologically, the disease is usually more severe in the mid-thoracic region than in the cervical spinal cord [20,21]. Most published series on VM describe a predominant involvement of the lateral and posterior columns of the spinal cord [20, 22]; however, in a pathologic study on 20 patients with VM, no significant longitudinal gradient was found in score severity in the posterior, lateral, or anterior columns [21]. Vacuoles are the result of edematous swelling within myelin, with splitting of the lamellae. The axons are usually normal until severe vacuolation occurs, followed by secondary Wallerian degeneration and axonal disruption [16, 18, 19]. The pathogenesis of VM is unknown, but is thought to be related to a combination of immune-mediated myelin, oligodendrocyte injury, and simultaneous impairment of repair mechanisms caused by a deficiency of the enzyme $S$ adenosylmethionine (SAM) [23, 24]. Published reports support an indirect relation between HIV and the pathogenesis of vacuolar myelopathy, peripheral neuropathy, and dementia. Because of the overlap between these syndromes, it is not completely clear whether we are dealing with disease entities or parts of a spectrum of tissue damage [1].

Due to the absence of randomized studies, there is no consensus on the treatment of HIVM. However, prelimi- nary data suggests that highly active antiretroviral therapy (HAART) has a positive effect on the clinical course of HIVM [25].Initial results on oral methionine supplementation for the treatment of HIVM were also promising [26]; however, according to a subsequent placebo-controlled study, there was no clinical benefit for patients who received methionine therapy [27]. Recently, intravenous immunoglobulin has been successfully used in a pilot study for the treatment of HIVM [28].

The diagnosis of VM is based on the clinical course and the exclusion of other causes of myelopathy. MR imaging findings of VM include bilaterally symmetrical increased signal intensity on T2-weighted MR images, predominantly located in the lateral and dorsal parts of the spinal cord [4, 5]. In one study, symmetrical, triangular, high-signal lesions were found within the gracile tract over several spinal segments, which correlated well with histopathological findings [5]. Since the inflammatory component is missing in VM, the blood-spinal cord barrier is not damaged, and no enhancement is present on post-contrast images. In a study of 21 patients with AIDS-associated myelopathy, spinal cord atrophy was the most common MR imaging finding ( $86 \%)$, followed by intrinsic cord hyperintensities on T2-weighted imaging (29\%) [29]; however, this study lacked pathologic correlation.

Many cases of VM may have been below the limits of resolution/detection of 1.0-1.5 T MRI units. With a highfield unit using optimized high resolution imaging acquisitions, the number and extent of intramedullary lesions detected on neuroimaging examinations will likely more closely approximate the actual number of cord lesions detected by the neuropathologist.

In the present study, performed at 3-T MR using DTI in asymptomatic HIV-positive patients, the results show that HIV-positive patients demonstrate changes in FA and MD, as well as the major and minor eigenvalues in the cervical spinal cord, compared to controls in the absence of abnormalities on conventional MR imaging. These changes consisted of decreased mean FA and increased mean MD and E1 to E3 in all ROIs. However, the differences did not reach statistical significance for FA, MD, E1, and E2. The only significant increase was found in the lateral ROI of the minor eigenvalue E3 in HIV-positive patients ( $p=0.013$; unpaired $t$-test).

In the spinal cord, the minor eigenvalues typically correspond to transverse diffusion, perpendicular to the longitudinal axis of the spinal cord. Animal studies have suggested that an increase in the minor eigenvalues occurs in the setting of demyelination, increased axonal diameter, and additional factors, including protein integrity [30]. The increased transverse diffusion seen in the normal-appearing spinal cord in HIV-positive patients suggests possible microscopic demyelination and could be an early sign of VM. The predominance of changes in DTI metrics in the 
lateral spinal cord, may reflect the distribution pattern of $\mathrm{VM}$, which has been found to be most severe in the lateral and posterior columns [20]. Taken into account that there was also a trend toward lower FA values $(p=0.073)$ and higher $\mathrm{MD}$, and E2 values $(p=0.063$ and $p=0.083$, respectively; unpaired $t$-test) in the lateral ROIs of HIVpositive patients compared to controls the measurement of lateral ROIs seems to be most promising for the early detection of HIVM.

VM is predominantly present in immunocompromised patients with low CD4 cell counts; however, one case of a patient with recurrent VM and CD4 cell count of 560/ml was recently reported [31]. In our study population, ten patients had CD4 cell counts of less than $200 / \mathrm{mm}^{3}$ and therefore can be considered to have AIDS [11]; however, the FA values of patients with and without AIDS did not show any significant difference.

The changes in the diffusion properties of the human spinal cord in subjects of different ages were studied and measured in a recently published study on 42 healthy volunteers [32]. The FA, the MD, and eigenvalues of the cervical spinal cord were compared for ROI- and diffusion tensor tractography (DTT)-based segmentation methods. The results of that and similar studies suggest that, independent of the segmentation approach used, the diffusion characteristics are age-dependent. In our study, patients and healthy subjects were age-matched; therefore, any age-related bias in our results can be excluded.

No association between VM and HIV myelitis or opportunistic infections of the CNS was found in one neuropathological study of 74 cases of VM in AIDS patients [33]. A close association with HIV encephalitis was found, which occurred in $80 \%$ of severe VM, $33 \%$ of moderate VM, and $17 \%$ of mild VM [33]. In our limited series, no patient had clinical or radiological signs of an HIV-related brain abnormality.

Our study has several limitations. First, the number of patients was relatively small. It might be possible that by inclusion of a larger study population the differences in the lateral ROIs of all DTI metrics would become significantly and that the measurement of the lateral columns subsequently could be useful as an early marker for HIVM.

Another potential limitation is one which is a challenge when acquiring DTI data of the spinal cord. We had experimented with various navigator sequences and also attempted cardiac gating to overcome some of the issues with CSF pulsation. However, when cardiac gating, the acquisition times became prohibitively long in terms of the data then suffering from respiratory and swallowing artefact. As a compromise, we utilized parallel imaging to reduce the acquisition time and reduce the gross motion. Newer sequences and techniques will allow us to acquire DTI data in a motion pulsatile cord as well as overcome some of the limitations with field inhomogeneity and Nyquist artifact. Another technical limitation is the ability to acquire DTI data from the thoracic spinal cord. The homogeneity and better $\mathrm{S} / \mathrm{N}$ in the volume neck coil for cervical cord DTI makes for superior data reproducibility than the use of the conventional phased array surface spine coil in the thoracic region. Therefore, we performed DTI of the cervical cord instead of the thoracic spinal cord although, according to the literature the pathology in VM seems to start in the mid-low thoracic cord, with cervical involvement as the disease becomes more severe [21]. Again, with improvements in sequence development and coil technology, this will be less of a limitation.

In addition, we included only asymptomatic HIV-positive patients, who may not have severe changes in the spinal cord. In an autopsy-based, case-control study, $46.5 \%$ of spinal cord specimens showed changes consistent with VM but only $26.8 \%$ of these cases had clinical symptoms of VM [2]. The presence of symptomatic myelopathy was related to the pathologic severity of the changes in the spinal cord [2].

It could be argued that the comparison of symptomatic instead of asymptomatic patients with healthy individuals could have revealed more differences in DTI. However, we decided against this approach for two reasons: first, patients with clinical symptoms of HIVM frequently show abnormalities already seen with conventional MR imaging, such as spinal cord T2-hyperintensities and atrophy [29], and DTI may not provide any additional clinically relevant information. Our main aim was to test a new method for the detection of subtle changes in the spinal cord that was superior to the currently routinely performed conventional MR imaging and therefore we decided to include asymptomatic HIV-positive patients only that could probably benefit from DTI.

Second, the number of HIV-positive patients with neurological disorders that can be attributed to HIVM has obviously decreased since the introduction of HAART [34], although controversies on the efficacy of HAART in the treatment of HIVM exist in the literature [35-37]. It is unknown if HAART has effectively reduced the incidence of vacuolization of the spinal cord or just mitigated the severity or progression of the demyelinating disease [38]. In this context, it would be interesting to examine patients with and without HAART to see if there is a difference, and to compare the DTI parameters with immunological and virological data. For further understanding of HIVM and its imaging findings, it would also be useful to include patients with HIV encephalopathy and to investigate the relationship between spinal cord and brain abnormalities.

To our knowledge, our study is the first to address DTI in HIVM, and further work regarding the use of this technique in symptomatic patients with and without abnormalities on conventional MR imaging and with HIV encephalopathy as well as in patients with and without HAART is required. 


\section{Conclusion}

DTI of the cervical spinal cord showed slightly decreased mean FA and increased mean MD, and E1 to E3 values in all ROIs in HIV-positive patients compared to healthy controls, but reached statistical significance in the lateral measurement of the minor eigenvalue E3 only. Our initial results therefore do not support the use of DTI for early detection of HIVM in asymptomatic HIV-positive patients; however, the fact that the differences in DTI metrics between patients and controls were most obvious in the lateral column of the cervical spinal cord could serve as a starting point for further DTI studies on HIVM.

Conflict of interest We declare that we have no conflict of interest.

Open Access This article is distributed under the terms of the Creative Commons Attribution Noncommercial License which permits any noncommercial use, distribution, and reproduction in any medium, provided the original author(s) and source are credited.

\section{References}

1. Bergmann M, Gullotta F, Kuchelmeister K, Masini T, Angeli G (1993) AIDS-myelopathy. A neuropathological study. Pathol Res Pract 189:58-65

2. Dal Pan GJ, Glass JD, McArthur JC (1994) Clinicopathologic correlations of HIV-1-associated vacuolar myelopathy: an autopsy-based case-control study. Neurology 44:2159-2164

3. Di Rocco A, Simpson DM (1998) AIDS-associated vacuolar myelopathy. AIDS Patient Care STDs 12:457-461

4. Thurnher MM, Post MJ, Jinkins JR (2000) MRI of infections and neoplasms of the spine and spinal cord in 55 patients with AIDS. Neuroradiology 42:551-563

5. Sartoretti-Schefer S, Blattler T, Wichmann W (1997) Spinal MRI in vacuolar myelopathy, and correlation with histopathological findings. Neuroradiology 39:865-869

6. Demir A, Ries M, Moonen CT, Vital JM, Dehais J, Arne P, Caille JM, Dousset V (2003) Diffusion-weighted MR imaging with apparent diffusion coefficient and apparent diffusion tensor maps in cervical spondylotic myelopathy. Radiology 229:37-43. doi:10.1148/radiol.2291020658229/1/37

7. Facon D, Ozanne A, Fillard P, Lepeintre JF, Tournoux-Facon C, Ducreux D (2005) MR diffusion tensor imaging and fiber tracking in spinal cord compression. AJNR Am J Neuroradiol 26:15871594. doi:26/6/1587

8. Shanmuganathan K, Gullapalli RP, Zhuo J, Mirvis SE (2008) Diffusion tensor MR imaging in cervical spine trauma. AJNR Am J Neuroradiol 29:655-659. doi:10.3174/ajnr.A0916

9. Hesseltine SM, Law M, Babb J, Rad M, Lopez S, Ge Y, Johnson G, Grossman RI (2006) Diffusion tensor imaging in multiple sclerosis: assessment of regional differences in the axial plane within normal-appearing cervical spinal cord. AJNR Am J Neuroradiol 27:1189-1193. doi:27/6/1189

10. Ohgiya Y, Oka M, Hiwatashi A, Liu X, Kakimoto N, Westesson PL, Ekholm SE (2007) Diffusion tensor MR imaging of the cervical spinal cord in patients with multiple sclerosis. Eur Radiol 17:2499-2504. doi:10.1007/s00330-007-0672-4

11. (1992) 1993 revised classification system for HIV infection and expanded surveillance case definition for AIDS among adolescents and adults. MMWR Recomm Rep 41:1-19

12. Mamata H, Jolesz FA, Maier SE (2005) Apparent diffusion coefficient and fractional anisotropy in spinal cord: age and cervical spondylosis-related changes. J Magn Reson Imaging 22:38-43. doi:10.1002/jmri.20357

13. Melhem ER (2000) Technical challenges in MR imaging of the cervical spine and cord. Magn Reson Imaging Clin N Am 8:435452

14. Maier SE, Mamata H (2005) Diffusion tensor imaging of the spinal cord. Ann NY Acad Sci 1064:50-60. doi:10.1196/ annals. 1340.011

15. Rossi C, Boss A, Lindig TM, Martirosian P, Steidle G, Maetzler W, Claussen CD, Klose U, Schick F (2007) Diffusion tensor imaging of the spinal cord at 1.5 and 3.0 Tesla. Rofo 179:219224. doi:10.1055/s-2007-962832

16. Henin D, Smith TW, De Girolami U, Sughayer M, Hauw JJ (1992) Neuropathology of the spinal cord in the acquired immunodeficiency syndrome. Hum Pathol 23:1106-1114

17. Goldstick L, Mandybur TI, Bode R (1985) Spinal cord degeneration in AIDS. Neurology 35:103-106

18. Gray F, Geny C, Lionnet F, Dournon E, Fenelon G, Gherardi R, Poirier J (1991) Neuropathologic study of 135 adult cases of acquired immunodeficiency syndrome (AIDS). Ann Pathol 11:236-247

19. Artigas J, Grosse G, Niedobitek F (1990) Vacuolar myelopathy in AIDS. A morphological analysis. Pathol Res Pract 186:228-237

20. Petito CK, Navia BA, Cho ES, Jordan BD, George DC, Price RW (1985) Vacuolar myelopathy pathologically resembling subacute combined degeneration in patients with the acquired immunodeficiency syndrome. N Engl J Med 312:874-879

21. Tan SV, Guiloff RJ, Scaravilli F (1995) AIDS-associated vacuolar myelopathy. A morphometric study. Brain 118(Pt 5):1247-1261

22. Maier H, Budka H, Lassmann H, Pohl P (1989) Vacuolar myelopathy with multinucleated giant cells in the acquired immune deficiency syndrome (AIDS). Light and electron microscopic distribution of human immunodeficiency virus (HIV) antigens. Acta Neuropathol 78:497-503

23. Tan SV, Guiloff RJ (1998) Hypothesis on the pathogenesis of vacuolar myelopathy, dementia, and peripheral neuropathy in AIDS. J Neurol Neurosurg Psychiatry 65:23-28

24. Di Rocco A, Bottiglieri T, Werner P, Geraci A, Simpson D, Godbold J, Morgello S (2002) Abnormal cobalamin-dependent transmethylation in AIDS-associated myelopathy. Neurology 58:730-735

25. Bizaare M, Dawood H, Moodley A (2008) Vacuolar myelopathy: a case report of functional, clinical, and radiological improvement after highly active antiretroviral therapy. Int J Infect Dis 12:442444. doi:10.1016/j.ijid.2007.09.014

26. Di Rocco A, Tagliati M, Danisi F, Dorfman D, Moise J, Simpson DM (1998) A pilot study of L-methionine for the treatment of AIDS-associated myelopathy. Neurology 51:266-268

27. Di Rocco A, Werner P, Bottiglieri T, Godbold J, Liu M, Tagliati M, Scarano A, Simpson D (2004) Treatment of AIDS-associated myelopathy with L-methionine: a placebo-controlled study. Neurology 63:1270-1275. doi:63/7/1270

28. Cikurel K, Schiff L, Simpson DM (2009) Pilot Study of Intravenous Immunoglobulin in HIV-Associated Myelopathy. AIDS Patient Care STDs. doi:10.1089/apc.2008.001810.1089/ apc. 2008.0018

29. Chong J, Di Rocco A, Tagliati M, Danisi F, Simpson DM, Atlas SW (1999) MR findings in AIDS-associated myelopathy. AJNR Am J Neuroradiol 20:1412-1416 
30. Schwartz ED, Cooper ET, Chin CL, Wehrli S, Tessler A, Hackney DB (2005) Ex vivo evaluation of ADC values within spinal cord white matter tracts. AJNR Am J Neuroradiol 26:390-397. doi:26/ 2/390

31. Anneken K, Fischera M, Evers S, Kloska S, Husstedt IW (2006) Recurrent vacuolar myelopathy in HIV infection. J Infect 52: e181-e183. doi:10.1016/j.jinf.2005. 08.023

32. Van Hecke W, Leemans A, Sijbers J, Vandervliet E, Van Goethem J, Parizel PM (2008) A tracking-based diffusion tensor imaging segmentation method for the detection of diffusion-related changes of the cervical spinal cord with aging. J Magn Reson Imaging 27:978-991. doi:10.1002/jmri.21338

33. Petito CK, Vecchio D, Chen YT (1994) HIV antigen and DNA in AIDS spinal cords correlate with macrophage infiltration but not with vacuolar myelopathy. J Neuropathol Exp Neurol 53:86-94
34. Yoritaka A, Ohta K, Kishida S (2007) Prevalence of neurological complications in Japanese patients with AIDS after the introduction of HAART. Rinsho Shinkeigaku 47:491-496

35. Aboulafia DM, Taylor L (2002) Vacuolar myelopathy and vacuolar cerebellar leukoencephalopathy: a late complication of AIDS after highly active antiretroviral therapy-induced immune reconstitution. AIDS Patient Care STDs 16:579-584. doi:10. 1089/108729102761882116

36. Oksenhendler E, Ferchal F, Cadranel J, Sauvageon-Martre H, Clauvel JP (1990) Zidovudine for HIV-related myelopathy. Am J Med 88:65 N-66 N

37. Staudinger R, Henry K (2000) Remission of HIV myelopathy after highly active antiretroviral therapy. Neurology 54:267-268

38. Di Rocco A (2007) HIV/AIDS and the nervous system. Elsevier. 